Journal Club

Editor's Note: These short, critical reviews of recent papers in the Journal, written exclusively by graduate students or postdoctoral fellows, are intended to summarize the important findings of the paper and provide additional insight and commentary. For more information on the format and purpose of the Journal Club, please see http://www.jneurosci.org/misc/ifa_features.shtml.

\title{
Dissociating the Effects of Past and Future on Neural Encoding of Sequences in The Hippocampus
}

\author{
Michael M. Yartsev \\ Department of Neurobiology, PhD Program, Weizmann Institute of Science, Rehovot 76100, Israel \\ Review of Ji and Wilson (http://www.jneurosci.org/cgi/content/full/28/18/4679)
}

When going about our daily business, we often dig into our memories to better foresee the outcomes of our behavior and plan future actions. One can think of such episodes as consisting of sequences of events, starting in the past and ending in the future. The hippocampus is believed to play a crucial role in our ability to learn sequences of events. This idea is supported by theoretical models, by data from healthy humans and patients with hippocampal lesions, and by lesion studies in rats (Eichenbaum and Cohen, 2001; Fortin et al., 2002). The neural code for sequence learning in the rat hippocampus has often been studied using a spatial alternation task on a figure-eight maze, in which the rat is required to alternate between the left and right sides of the maze while repeatedly crossing the central stem (Fig. $1 A$, blue).

Previous studies using the figure-eight maze have revealed a striking phenomenon: hippocampal "place cells," neurons that generally tend to discharge at a particular location in the environment (the "place field"), became activated in a trajectory-selective manner that can be viewed as a spatial sequence. That is, many

Received May 30, 2008; revised June 26, 2008; accepted June 26, 2008. M.M.Y is supported by the Feinberg Graduate School of the Weizmann Institute of Science. I thank N. Ulanovsky and M. Melcón for helpful comments on this manuscript.

Correspondence should be addressed to Michael M. Yartsev, Department of Neurobiology, Weizmann Institute of Science, Rehovot 76100, Israel. E-mail:myartsev@weizmann.ac.il.

D0I:10.1523/JNEUROSCI.2442-08.2008

Copyright $\odot 2008$ Society for Neuroscience $\quad$ 0270-6474/08/288383-02\$15.00/0 neurons discharged only when the animal ran the trajectory $\mathrm{R}_{\text {pre }} \rightarrow$ stem $\rightarrow \mathrm{L}_{\text {post }}$, whereas other neurons discharged only on the trajectory $\mathrm{L}_{\text {pre }} \rightarrow$ stem $\rightarrow \mathrm{R}_{\text {post }}$ (Fig. $1 \mathrm{~A}$, blue) (Wood et al., 2000; Lee et al., 2006). However, this alternation design did not allow determining whether the sequential encoding is a result of the effect of the past or the future, because past and future trajectories were always coupled: $\mathrm{R}_{\text {pre }}$ was always followed by $\mathrm{L}_{\text {post }}$ and $\mathrm{L}_{\text {pre }}$ was always followed by $\mathrm{R}_{\text {post }}$ (Fig. 1, blue).

A recent study by Ji and Wilson (2008) published in The Journal of Neuroscience introduced a new twist to the figure-eight maze task. Animals were trained to gradually alter their previously established figure-eight routes into novel circular routes (Fig. $1 A$, red), so it was possible to fill in the contingency table of possible sequences (Fig. $1 B$ ) and dissociate the effects of past and future.

The study by Ji and Wilson (2008) contains three major findings. First, the authors observed a strong dependency of hippocampal activity on past, but not future, trajectories: neural firing was strongly correlated between trajectories sharing the same past, but not between trajectories sharing the same future [Ji and Wilson (2008), their Fig. 2 (http://www. jneurosci.org/cgi/content/full/28/18/ $4679 / F 2)]$. Second, because the rats gradually changed their old trajectories to new ones, the authors could correlate the dynamic change in neural activity with the dynamics of behavioral changes. They found that changes in neural activity pre- ceded the behavioral change [Ji and Wilson (2008), their Fig. 3 (http://www. jneurosci.org/cgi/content/full/28/18/ 4679/F3)] and then continued to evolve, becoming gradually more similar for samepast trajectories and more dissimilar for different-past trajectories [Ji and Wilson (2008), their Fig. 5 (http://www.jneurosci. org/cgi/content/full/28/18/4679/F5)]. Third, unlike previous studies that examined only changes in place fields on the central stem (Wood et al., 2000), the dynamic changes in firing rate were observed also for place fields extending beyond the stem. Interestingly, the dependence on the past was found only in place fields adjacent to the stem, and the effect diminished with increasing distance from the stem [Ji and Wilson (2008), their Fig. 8 (http://www.jneurosci. org/cgi/content/full/28/18/4679/F8)].

In turning to discuss the methodology, results, and our interpretations of the study by Ji and Wilson (2008), we will focus on four topics. First, we begin with the most fundamental issue concerning the interpretation of the results and consider whether the task used by Ji and Wilson (2008) is a hippocampus-dependent task, requiring the computations performed by the hippocampus. Both spatial and nonspatial sequence learning tasks involving a delay have been shown to be hippocampus dependent (Fortin et al., 2002; Ferbinteanu and Shapiro, 2003). Importantly, a recent study using a similar task to that used by Ji and Wilson (2008) showed that rats with bilateral hippocampal lesions 
suffer no deficit in performing this task unless a delay was introduced between trials, in which case task performance gradually degraded with increasing delay (Ainge et al., 2007). Thus, it is plausible that in the study by Ji and Wilson (2008), which did not use any delay, the acquisition and performance of the behavioral task reflected processing upstream of the hippocampus (Ainge et al., 2007), rather then processing within the hippocampus itself.

Second, the details of the preliminary training procedures are noteworthy, because recent studies using a similar task to that of Ji and Wilson (2008) have shown that specific aspects of preliminary training (e.g., training with or without barriers) can substantially affect the robustness of trajectory-dependent representation in the hippocampus (Wood et al., 2000; Lee et al., 2006; Ainge et al., 2007). For example, one study did not find even a single hippocampal cell to be dependent on past or future trajectories when barriers were not used during initial training, in striking contrast to when barriers were used (Bower et al., 2005). In the study of Ji and Wilson (2008), no barriers were used in any stage of the training (D. Ji, personal communication), yet surprisingly, a robust trajectory dependence was found. Understanding this apparent discrepancy in the effects of barriers on trajectorydependent hippocampal encoding would require further experimentation.

Third, the authors did not find a forward shift in place-field locations on the figure-eight maze, in contrast to recent studies (Lee et al., 2006). Ji and Wilson (2008) proposed that differences in experimental procedures underlie this difference in neural dynamics. However, an alternative interpretation is that the reward location was the dominant factor. Hippocampal dynamics have been shown to encode goal-directed behavior (Shapiro et al., 2006); hence, the reward locations, rather then the animal's direction of motion (forward or backward), may determine the direction of place-field shift. Indeed, in the Lee et al. (2006) design, reward was located near the end of the stem, and a forward shift was observed, whereas Ji and Wilson (2008) placed the reward close to the beginning of the stem, which might have prevented the forward

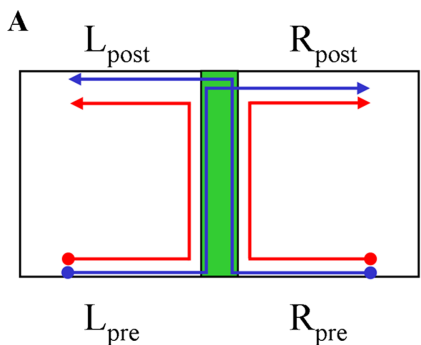

$$
\begin{aligned}
& \mathrm{R}_{\text {pre }} \rightarrow \text { Stem } \rightarrow \mathrm{L}_{\text {post }} \\
& \mathrm{L}_{\text {pre }} \rightarrow \text { Stem } \rightarrow \mathrm{R}_{\text {post }} \\
& \mathrm{R}_{\text {pre }} \rightarrow \text { Stem } \rightarrow \mathrm{R}_{\text {post }} \\
& \mathrm{L}_{\text {pre }} \rightarrow \text { Stem } \rightarrow \mathrm{L}_{\text {post }}
\end{aligned}
$$

B

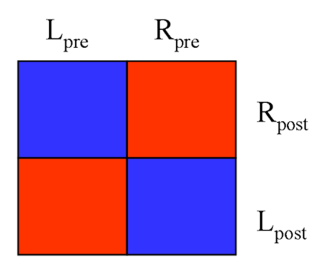

Figure 1. Properties of the standard and modified figure-eight design. $\boldsymbol{A}$, Schematic of a figure-eight maze. The standard spatial alternation design (blue) cannot dissociate the effects of past and future, as these are always coupled. The two additional trajectories added to the task by Ji and Wilson (2008) (in red) enabled double dissociation between the effects of past and future. $\boldsymbol{B}$, Contingency table showing the case of the standard alternation task (blue) and added components of the modified circular task (red). $R_{\text {pre }}$ and $L_{\text {pre }}$ designate the right and left sides, respectively, of the maze before the animal enters the stem (colored green in $\boldsymbol{A}$ ), and $\mathrm{R}_{\text {post }}$ and $\mathrm{L}_{\text {post }}$ are the right and left sides, respectively, of the maze after the animal exits the stem.

shift of place fields and is in fact in agreement with the backward shift in placefield locations reported by the authors.

Finally, we consider the notion that hippocampal neurons are affected strictly by past trajectories, as suggested by the results of Ji and Wilson (2008). An interesting recent study found that, in fact, ensembles of hippocampal neurons predicted future trajectories at important decision points (Johnson and Redish, 2007). Importantly, this prediction of the future occurred on timescales of tens or hundreds of milliseconds, much shorter than the timescale of the representation of the past in the study by Ji and Wilson (2008), which was on the order of seconds or more. This suggests that the effects of past and future on hippocampal neural activity might operate on different timescales. Thus, one could hypothesize that when analyzing the data on long timescales, one would find primarily effects of the past, as in the study by Ji and Wilson (2008), whereas analysis on shorter timescales would reveal primarily effects of future planning, as in the study by Johnson and Redish (2007).

Together, the results presented by Ji and Wilson (2008) add important insight to our understanding of the underlying neural code for novel sequence learning in the hippocampus and the effects of past and future events. Importantly, a central future goal for these types of studies will be to categorize which tasks are hippocampus dependent and which are not to better relate the neural activity to actual hippocampal function. In addition, it will be important to elucidate the exact aspects of the task that are relevant for encoding particular parts of episodes by the hippocampus. This challenge will go hand in hand with our understanding of how past and future events influence the hippocampal neural code.

\section{References}

Ainge JA, van der Meer MA, Langston RF, Wood ER (2007) Exploring the role of contextdependent hippocampal activity in spatial alternation behavior. Hippocampus 17: 988-1002.

Bower MR, Euston DR, McNaughton BL (2005) Sequential-context-dependent hippocampal activity is not necessary to learn sequences with repeated elements. J Neurosci 25: 1313-1323.

Eichenbaum H, Cohen NJ (2001) From conditioning to conscious recollection: memory systems of the brain. New York: Oxford UP.

Ferbinteanu J, Shapiro ML (2003) Prospective and retrospective memory coding in the hippocampus. Neuron 40:1227-1239.

Fortin NJ, Agster KL, Eichenbaum HB (2002) Critical role of the hippocampus in memory for sequences of events. Nat Neurosci 5:458-462.

Ji D, Wilson MA (2008) Firing rate dynamics in the hippocampus induced by trajectory learning. J Neurosci 28:4679-4689.

Johnson A, Redish AD (2007) Neural ensembles in CA3 transiently encode paths forward of the animal at a decision point. J Neurosci 27:12176-12189.

Lee I, Griffin AL, Zilli EA, Eichenbaum H, Hasselmo ME (2006) Gradual translocation of spatial correlates of neuronal firing in the hippocampus toward prospective reward locations. Neuron 51:639-650.

Shapiro ML, Kennedy PJ, Ferbinteanu J (2006) Representing episodes in the mammalian brain. Curr Opin Neurobiol 16:701-709.

Wood ER, Dudchenko PA, Robitsek RJ, Eichenbaum H (2000) Hippocampal neurons encode information about different types of memory episodes occurring in the same location. Neuron 27:623-633. 